\title{
Development of a GUI for automating quality control of dose calibrators using Python
} \author{
Elkhoukhi ${ }^{4}$ \\ ${ }^{1}$ Ibn Tofail University, Laboratory of Physics Materials and Subatomic, Kenitra, Morocco \\ ${ }^{2}$ CNESTEN, Department of radiopharmaceutical production, Rabat, Morocco \\ ${ }^{3}$ Abdelmalk Essaadi University, ERESEN, Tetouan, Morocco \\ ${ }^{4}$ CNESTEN, Department of Safety and Security, Rabat, Morocco
}

Meryeme Bellahsaouia ${ }^{1, *}$, Mohammed Rabie Bricha ${ }^{2}$, Omaima Essaad Belhaj ${ }^{3}$, El Mahjoub Chakir ${ }^{1}$, Hamid $_{\text {Boukhal }}{ }^{3}$, Taher

\begin{abstract}
Quality control of dose calibrators is essential to evaluate the accuracy of the instrument response. In this work, a GUI (Graphical User Interface) has been developed to facilitate performing and recording quality control tests of dose calibrators. The interface is capable to automate several tests which include routine checks, accuracy test, linearity test, reproducibility test, repeatability test, concordance $\mathrm{MBq} / \mathrm{mCi}$ test, and geometry test. In principle, the program computes correction factors that should be applied to minimize the uncertainty of measurements and the determining factors for success or failure of each test, then visualizes the results as tables and curves into a pdf file. Therefore, this interface can be considered as an efficient tool for performing quality control tests of dose calibrators thought it is still unable to offer the correction factors for the geometry test without experiment which will be achieved by integrating Monte Carlo simulation into the GUI.
\end{abstract}

Keywords: quality control tests, dose calibrator, ionization chamber, correction factors, GUI development.

\section{Introduction}

Dose calibrators (DC) are widely used in nuclear medicine to assay the activity of a radioactive source administered to the patient in a diagnostic or therapeutic process. The objective of this assay is to assure that the patient receives the minimum absorbed dose compatible with obtaining a high-quality diagnostic image or with achieving a desired therapeutic outcome. This instrument is a well-type ionization chamber filled with pressurized gas. When radiation emitted by the assayed radionuclide was absorbed into the gas, an ion pair will be created and collected with two electrodes maintained at voltage difference. The produced current will be amplified and measured by an electrometer [1]. The displayed response on the screen represents the source activity after the conversion of measured current using a calibration coefficient which is critical in the instrument performance assurance: an appropriate calibration coefficient should be associated with a particular radionuclide in a specific source geometry since the response of DC depend on several factors such as the type and the energy of radiation emitter, the source activity, the source geometry, and the source position [2].

Unfortunately, manufacturers supply calibration coefficient for a specific number of sources, thus, the calculation of other sources' correction factors is indispensable to assure the accuracy of the DC response. This task can be performed using various methods such as Monte Carlo Method and some experimental methods that should be selected in accordance with the accuracy required for the assay $[3,4,5]$. The significance of these factors depends strongly on the radionuclide type: studies suggest that the beta emitters are more sensitive to the container material, as the self-absorption phenomenon is more important for this type of radiation [6, 7, 8]. Moreover, the efficiency for those radionuclides increases with the energy [9]. For gamma emitters, the uncertainty due to the geometry factor is inversely proportional to the source energy. The relative response increases when the horizontal position of the source is farther away from the standard position for high and medium energy, whilst it decreases for low energy emitters [5]. For the volume effect, several studies observed that the significance of the deviation of measurements increases with the filling volume, which can be explained by the variation of the angle solid that determines the available sensitive volume for detection $[5,6,10]$. In summary, the variation of one of those factors mentioned previously can greatly affect the reading activity. Hence, it is recommended to perform quality control tests regularly in order to update those factors and, consequently, provide an adequate result for the activity assayed.

\footnotetext{
*Corresponding author:_meryemebellahsaouia@gmail.com
} 
Avoiding any overestimate or underestimate of the dose administered to the patient is vital to achieving the principles of radiological protection that were implemented to protect the person and the environment against the harmful effects of ionizing radiations [11]. These principles involve the justification which imposes that the benefit expected (the diagnostic information or the therapeutic effect) must exceed the risk associated with the radiation exposure, the optimization which requires a balance between the administered activity and the desired effect of the exposure, and the limitation of dose which states that the absorbed dose should remain less than the limits recommended by the ICRP (International Commission on Radiological Protection) [12].

Because of the widespread influence of the estimating of the true activity on the public health and the environment, quality control tests of dose calibrators were established. Manufacturers must provide procedures for all tests that should be performed as well as the testing frequency. Moreover, the International Atomic Energy Agency (IAEA) has given recommendations on performing routine activity measurements and quality control testing that include voltage check, zero adjust, background test, constancy, accuracy test, linearity test, reproducibility test, and geometry test [13]. The aim of this work is to develop a GUI enabling to automate a common quality control testing of dose calibrator. The GUI has been built using python programming language that includes all necessary libraries for carrying out requisite operations related to those tests. In this paper, the working process of the GUI will be described and quality control testing will be applied to a MEDI404 dose calibrator as an example.

\section{Materials and Methods}

\subsection{The program structure}

The GUI was created using python 3.8 that required multiple libraries. The first one is PyQt5 which was used to build the GUI. For carrying out mathematical operations, statistics and math modules were called. Pandas, an open source Python package, was used to analyze data gathered in several tests. Data can be saved as csv files (Comma Separated Values file) using os and csv libraries then plotted by matplotlib (a comprehensive library for creating static, animated, and interactive visualizations in Python). Finally, reportlab library was called to create a pdf file that has combined all results.

The program was constituted of five classes: a class for the main window building, three classes for making modal dialogs, and the last one to create a canvas that figures render into. Each of these classes involves its own methods which can be divided into five groups depending on its functions. The first group gets information entered by the user, stores it in variables, and saves it into files. The second extracts data from files and displays it on the screen. Two principal groups help to obtain results, one for analyzing data and the other to visualize it on graphs. The last group calls all variables, data, and figures and assembles them into one file.

\subsection{Quality control tests}

The GUI permits the user to perform common tests of DC which involve routine checks (high voltage, current check, zero adjust, and contamination check), accuracy test, linearity test, reproducibility test, repeatability test, concordance $\mathrm{MBq} / \mathrm{mCi}$ test, and geometry test. To highlight the working process of this tool, a MEDI404 dose calibrator was used for carrying out some of these tests. The ${ }^{57} \mathrm{Co},{ }^{137} \mathrm{Cs}$ and ${ }^{99 \mathrm{~m}} \mathrm{Tc}$ sources were utilized in accuracy test, linearity test, and reproducibility test.

\subsubsection{Routine checks}

In these tests, the user should give parameter values associated with each test to be compared with the manufacturer tolerance which can be modified according to the DC model.

The parameter entered in the voltage test is the polarizing voltage of the ionization chamber while the current test parameter is related to the background [14]. For zero adjust, two parameters that compensate for the amplifier deviation should be provided [15]. Table 1 presents default values of tolerance limits for these tests in the program.

Table 1: Default values of tolerance limits for voltage check, current check, and zero adjust.

\begin{tabular}{|c|c|c|}
\hline test & parameter & Tolerance range \\
\hline High voltage & $\begin{array}{c}\text { Chamber voltage } \\
(\mathrm{V})\end{array}$ & $135-165$ \\
\hline Current check & Bias current (pA) & $0.05-0.15$ \\
\hline \multirow{2}{*}{ Zero adjust } & Preamplifier gain & $3100-4500$ \\
\cline { 2 - 3 } & I-to-Ub-factor & $3100-4500$ \\
\hline
\end{tabular}

The contamination check is established to assure that the instrument is not contaminated: measurements with and without the sample holder are taken in the absence of the radioactive source, and the difference between them must be less than $100 \mathrm{kBq}$.

The success or failure of each test can be determined by pressing the button "Go" and the results that include parameter value and decision taken for each test will be assembled into a table and displayed on the part "result" of the GUI (Fig.1).

\subsubsection{Accuracy test}

This test is designed to ensure the stability of the DC response. Long half-life solid sources whose energies 
cover the range of routine usage were used. ${ }^{57} \mathrm{Co},{ }^{137} \mathrm{Cs}$ and ${ }^{99 \mathrm{~m}} \mathrm{Tc}$ sources are commonly used to evaluate the DC response for low, medium, and high energy range respectively [2]. The measured activity of each source was compared with the reference activity supplied by a national or international standard radioactivity laboratory or from a secondary standard laboratory ${ }^{1}$. The relative bias $b$ should be with $\pm 5 \%$ after the decay correction [13].

$$
\begin{gathered}
b=\frac{A_{\text {ref }}-A_{m}}{A_{\text {ref }}} \times 100 \\
(S E Q \text { Equation } \backslash * A R A B I C 1)
\end{gathered}
$$

Where $A_{m}$ is the measured activity and $A_{\text {ref }}$ is the reference activity.

The developed program gets characteristics of each source and the activities values, calculates the correction factor $F_{C}$ and the relative bias $b$ to decide if the test has passed or not, then visualizes the results into a control chart. Input data are saved as csv files to be involved in the final report.

$$
F_{C}=\frac{A_{r e f}}{A_{m}}
$$

\subsubsection{Linearity test}

The linearity test evaluates linearity response of the instrument over the range of activity used in routine practices. There are three methods to perform this test: time decay method based on the decay phenomena to cover the entire activity range, shield method that depends on attenuation shields, and graded method that use samples with different activity [16]. The decay method is the most precise. It consists in measuring a short half-life source activity at regular intervals, covering about 10 half-lives, until the activity is less than $1 \mathrm{MBq}$ [13]. The relative standard deviation of bias between measured activity and reference activity should be less than 5\% [14].

The program calculates the reference activity $A_{\text {ref }}$ corrected for decay, the bias for each measurement, and the relative standard deviation of the calculated bias; then deduces the status of test which will be stated on the status bar.

$A_{\text {ref }}=A_{0} e^{-\lambda t}$

Where $A_{\text {ref }}$ is the reference activity at the time t, $A_{0}$ is the activity at the reference time and $\lambda$ is the decay constant.

\subsubsection{Reproducibility test}

The reproducibility test examines the deviation of a sequence of measurements from the average when measure conditions are changed. A series of measurements are taken using a long half-life source. The program calculates the average of measured activities $\underline{A}$ and the relative standard deviation RSD that must be less

\footnotetext{
${ }^{1}$ A laboratory providing "standardized radioactivity samples that has established measurement traceability to a national metrology institute" [13].
}

than $5 \%$, then shows the status of the test and plot a control chart that illustrates the distribution of the activity.

$$
R S D=\frac{\sqrt{\frac{\sum\left(A_{i}-\underline{A}\right)^{2}}{n-1}}}{\underline{A}} \times 100
$$

Where $\mathrm{n}$ is the number of measurements and $\mathrm{A}_{\mathrm{i}}$ the individual measured activity.

\subsubsection{Repeatability test}

In contrast to reproducibility, repeatability test should be performed without removing the source from the ionization chamber for each measurement. The rest of the process is similar to that of reproducibility.

\subsubsection{Correlation test $(\mathrm{MBq} / \mathrm{mCi})$}

As its name implies, this test confirms the concordance between measurement activity in $\mathrm{MBq}$ and $\mathrm{mCi}$. The ratio between them should remain constant and equal to 37 $\mathrm{MBq} / \mathrm{mCi}$. Once the results of several measurements in $\mathrm{MBq}$ and $\mathrm{mCi}$ were registered in the program, the conversion factor $\mathrm{F}_{\text {conv }}$ and its relative standard deviation RSD are calculated. If RSD is less than $1 \%$, the test is considered successful [14].

$$
\begin{aligned}
F_{\text {conv }} & =\frac{\text { Measured Activity in } M B q}{\text { Measured Activity in } m C i} \\
R S D & =\frac{\sigma}{37} \times 100
\end{aligned}
$$

Where $\sigma$ is the standard deviation of $F_{\text {conv }}$.

\subsubsection{Geometry test}

The geometry test is implemented to ensure that effects of container and sample volume are integrated into the reading activity. The procedure consists of measuring the sample activity in standard volume $A_{r e f}$, then diluting this activity with a fixed volume of water and assay it several times until the volume of the container is $67 \%$ full. The measured activity for each volume is compared with the activity $A_{\text {ref }}$, and a correction factor should be applied if the bias between them is more than $5 \%$ [1].

The GUI calculates correction factors related to the volume $F_{v}$ when the user enters the activity value of standard volume $A_{\text {ref }}$ and activity values of different volumes assayed $A_{i}$, then a graph that summarizes calculated correction factors will be displayed in the window.

$$
\begin{aligned}
& F_{v} \\
& =\frac{A_{\text {ref }}}{A_{i}} \\
& \backslash * A R A B I C 7)
\end{aligned}
$$

( SEQ Equation 
Fig.3.Accuracy test performing using the GUI.

\section{Results and Discussion}

Fig. 1 presents the main window of the GUI at startup. The user has to specify detector and sample characteristics, then he can choose the test he wants to perform. After entering data of such test and save it, the user can modify or even delete this data.

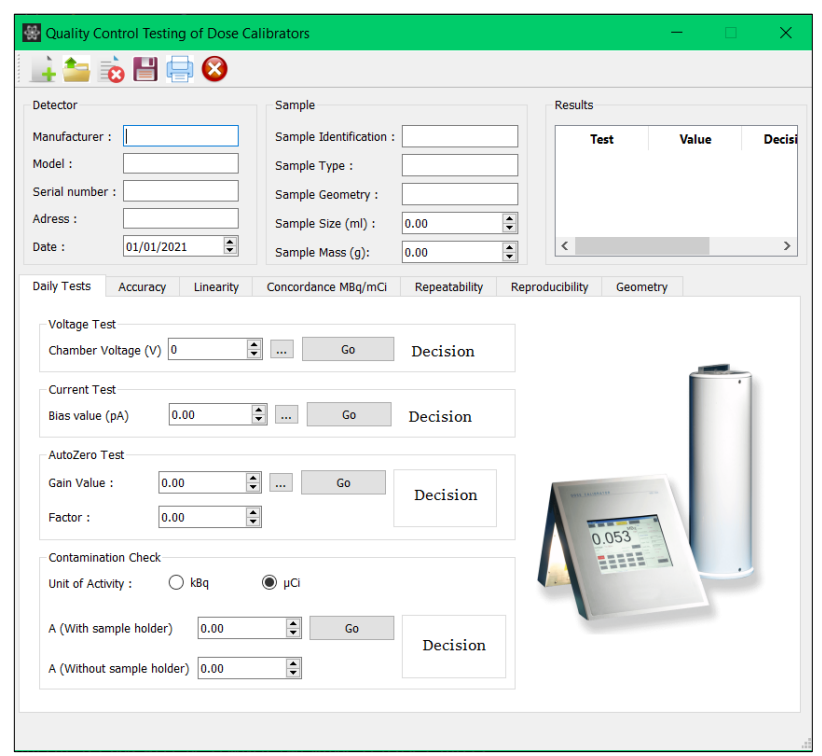

Fig.1.The main window of the GUI at startup.

Performing of routine checks, accuracy test, linearity test, and reproducibility test are set out in Fig.2, Fig.4, Fig.5, and Fig.6 respectively.

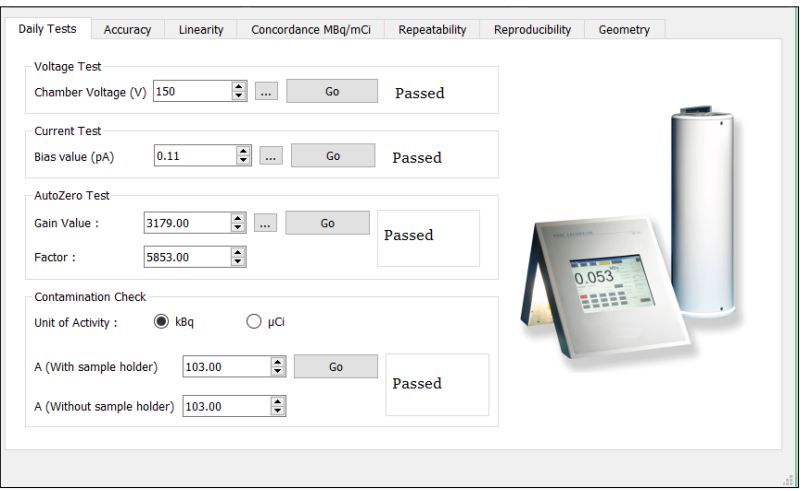

Fig.2.Daily checks performing using the GUI.

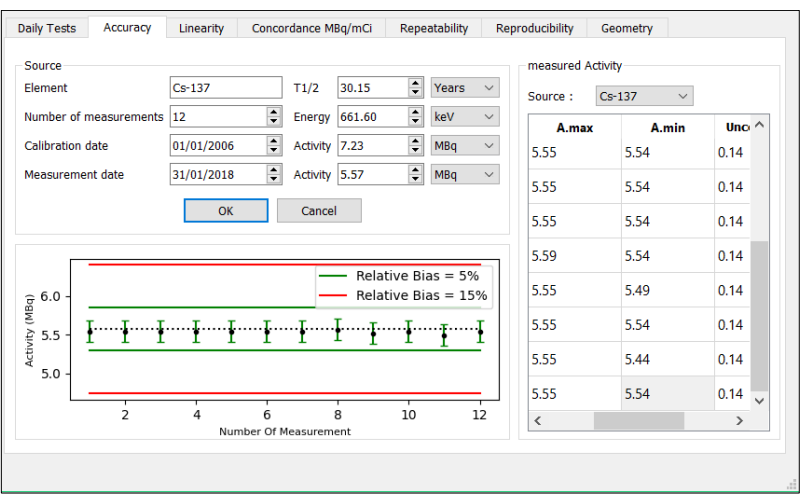

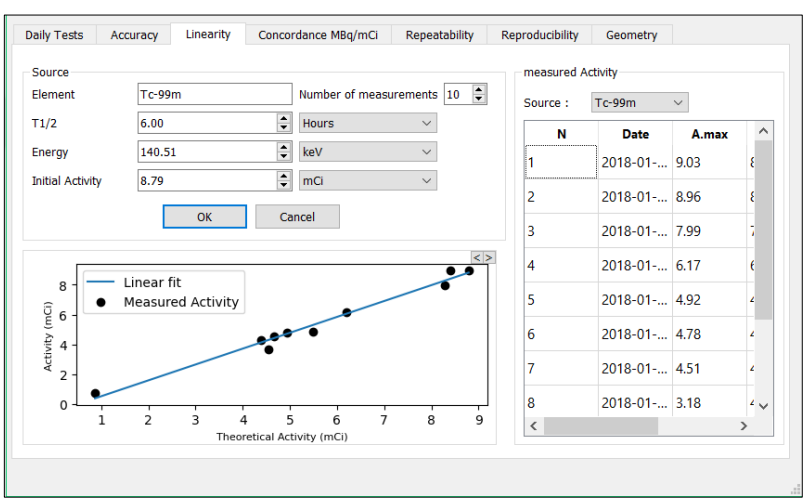

Fig.4.Linearity test performing using the GUI.

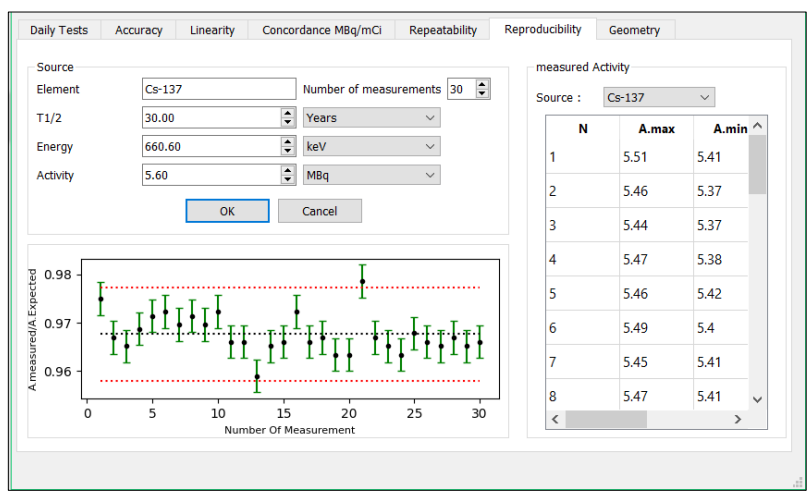

Fig.5.Reproducibility test performing using the GUI.

The report which includes data entered and tests outcome will appear if the user presses the icon "print". In the section of accuracy test, the resulting report contains a table that includes measured activity, expected activity, and bias between them for each measurement (Fig.6); a table that summarizes the test outcome (Fig.7); and control charts which reveal the tolerance range and the position of each measurement relative to this range (Fig.8).

\begin{tabular}{|c|c|c|c|c|c|}
\hline \multirow{2}{*}{$\begin{array}{c}\text { Number of } \\
\text { Measurement }\end{array}$} & \multicolumn{3}{|c|}{ Measured Activity (MBq) } & \multirow{2}{*}{$\begin{array}{c}\text { Theoretical } \\
\text { Activity (MBq) }\end{array}$} & Relative Bias (\%) \\
\hline & Maximum Activity & Minimum Activity & Average Activity \\
\hline 1.0 & 5.55 & 5.54 & 5.545 & 5.57 & 0.449 \\
\hline 2.0 & 5.55 & 5.54 & 5.545 & 5.57 & 0.449 \\
\hline 3.0 & 5.55 & 5.54 & 5.545 & 5.57 & 0.449 \\
\hline 4.0 & 5.55 & 5.54 & 5.545 & 5.57 & 0.449 \\
\hline 5.0 & 5.55 & 5.54 & 5.545 & 5.57 & 0.449 \\
\hline 6.0 & 5.55 & 5.54 & 5.545 & 5.57 & 0.449 \\
\hline 7.0 & 5.55 & 5.54 & 5.545 & 5.57 & 0.449 \\
\hline 8.0 & 5.59 & 5.54 & 5.565 & 5.57 & 0.09 \\
\hline 9.0 & 5.55 & 5.49 & 5.52 & 5.57 & 0.898 \\
\hline 10.0 & 5.55 & 5.54 & 5.545 & 5.57 & 0.449 \\
\hline 11.0 & 5.55 & 5.44 & 5.495 & 5.57 & 1.346 \\
\hline
\end{tabular}

Fig.6.Table that includes data of Cs-137 for accuracy test in the pdf file.

\begin{tabular}{|c|c|c|c|c|c|}
\hline Sources & $\begin{array}{c}\text { Measured } \\
\text { Activity (MBq) }\end{array}$ & $\begin{array}{c}\text { Expected } \\
\text { Activity (MBq) }\end{array}$ & Relative Bias (\%) & $\begin{array}{c}\text { Correction } \\
\text { Factor }\end{array}$ & Decisions \\
\hline Co-57 & 6.206 & 6.18 & -0.425 & 1.004 & Passed \\
\hline Cs-137 & 5.54 & 5.57 & 0.531 & 0.995 & Passed \\
\hline
\end{tabular}

Fig.7.Table that includes results of accuracy test in the pdf file. 


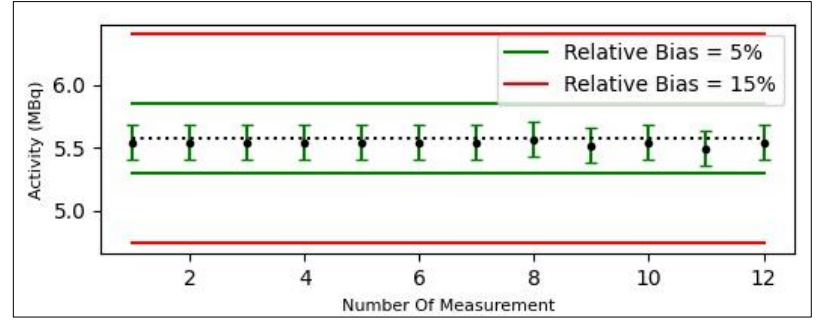

Fig.8.Control chart of Cs-137 for accuracy test plotted with the program.

Data of linearity test was combined in a table and visualized in a graph as it is show in Fig.9 and Fig.10. This data presents the activity of $99 m T c$ over time and the bias between measured activity and expected activity. Fig.11 is plotted to illustrate deviations of measured activity from the linear fitted curve and final results of the test are shown in a table at the end of the section (Fig.12).

\begin{tabular}{||c|c|c|c|c|c|c|c||}
\hline \multirow{2}{*}{ Date and Hour } & \multirow{2}{*}{ Elapsed Time } & \multicolumn{3}{|c|}{ Measured Activity $(\mathrm{mCl})$} & \multicolumn{2}{|c|}{ Expected Activity } & \multirow{2}{*}{ Bias } \\
\cline { 3 - 7 } & & Maximun & Minimun & Average & Dose $(\%)$ & Dose $(\mathrm{mCl})$ & \\
\hline 2018-01-31 14:15:00 & $0: 00: 00$ & 9.03 & 8.85 & 8.94 & 1.0 & 8.79 & 0.15 \\
\hline $2018-01-3114: 38: 00$ & $0: 23: 00$ & 8.96 & 8.92 & 8.94 & 0.957 & 8.409 & 0.531 \\
\hline $2018-01-3114: 46: 00$ & $0: 31: 00$ & 7.99 & 7.97 & 7.98 & 0.942 & 8.281 & 0.301 \\
\hline $2018-01-3117: 16: 00$ & $3: 01: 00$ & 6.17 & 6.1 & 6.135 & 0.706 & 6.204 & 0.069 \\
\hline $2018-01-3118: 20: 00$ & $4: 05: 00$ & 4.92 & 4.78 & 4.85 & 0.624 & 5.484 & 0.634 \\
\hline $2018-01-3119: 15: 00$ & $5: 00: 00$ & 4.78 & 4.73 & 4.755 & 0.561 & 4.933 & 0.178 \\
\hline $2018-01-3119: 45: 00$ & $5: 30: 00$ & 4.51 & 4.5 & 4.505 & 0.53 & 4.656 & 0.151 \\
\hline $2018-01-3119: 57: 00$ & $5: 42: 00$ & 3.18 & 4.1 & 3.64 & 0.518 & 4.55 & 0.91 \\
\hline
\end{tabular}

Fig.9.Table that includes data of linearity test in the pdf file.

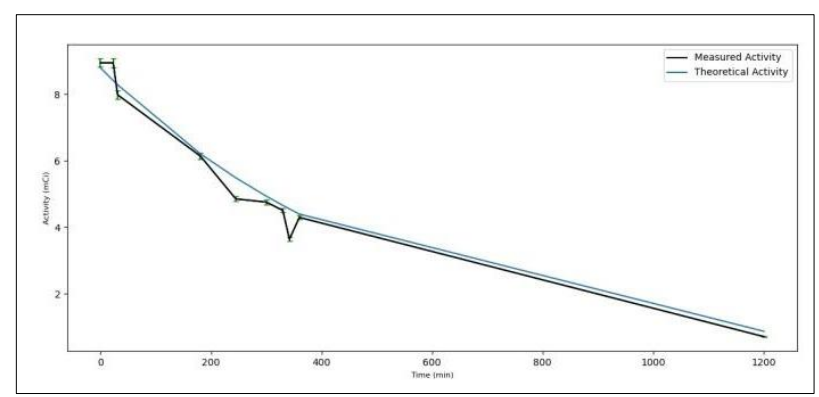

Fig.10.Activity decrease of $99 \mathrm{mTc}$ as a function of time for linearity test plotted with the program.

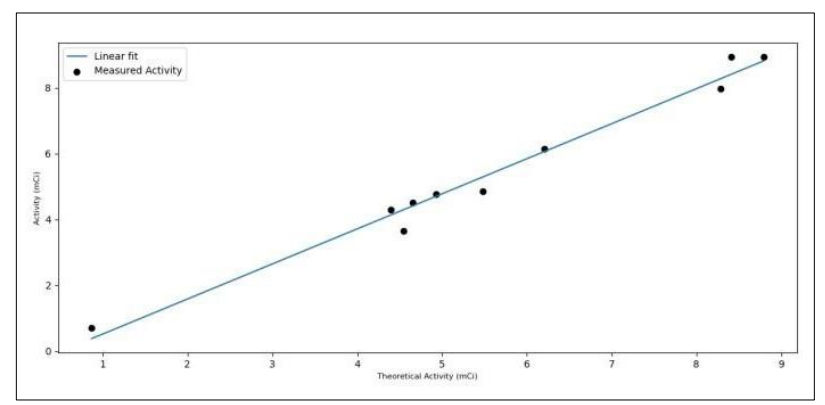

Fig.11.Linear regression curve between measured activity and expected activity for linearity test plotted with the program.

\begin{tabular}{|c|c|c|c|}
\hline Average Bias & $\begin{array}{c}\text { Relative Standard } \\
\text { Deviation }\end{array}$ & $\begin{array}{c}\text { Correlation } \\
\text { Coefficient }\end{array}$ & Decision \\
\hline 0.318 & 0.88 & 0.99 & Failed \\
\hline
\end{tabular}

Fig.12. Table that includes results of linearity test in the pdf file.

Results of the reproducibility test recorded on the pdf file are shown in Fig.13, Fig.14, and Fig.15. The control chart presents the activity of each measurement and the limits of acceptability for this test.

\begin{tabular}{|c|c|c|c|}
\hline \multirow{2}{*}{$\begin{array}{l}\text { Number of } \\
\text { Measurement }\end{array}$} & \multicolumn{3}{|c|}{ Measured Activity (MBq) } \\
\hline & Maximun Activity & Minimun Activity & Average Activity \\
\hline 1.0 & 5.51 & 5.41 & 5.46 \\
\hline 2.0 & 5.46 & 5.37 & 5.415 \\
\hline 3.0 & 5.44 & 5.37 & 5.405 \\
\hline 4.0 & 5.47 & 5.38 & 5.425 \\
\hline 5.0 & 5.46 & 5.42 & 5.44 \\
\hline 6.0 & 5.49 & 5.4 & 5.445 \\
\hline 7.0 & 5.45 & 5.41 & 5.43 \\
\hline 8.0 & 5.47 & 5.41 & 5.44 \\
\hline 9.0 & 5.45 & 5.41 & 5.43 \\
\hline 10.0 & 5.49 & 5.4 & 5.445 \\
\hline 11.0 & 5.44 & 5.38 & 5.41 \\
\hline 12.0 & 5.44 & 5.38 & 5.41 \\
\hline 13.0 & 5.37 & 5.37 & 5.37 \\
\hline 14.0 & 5.42 & 5.39 & 5.405 \\
\hline 15.0 & 5.44 & 5.38 & 5.41 \\
\hline 16.0 & 5.49 & 5.4 & 5.445 \\
\hline 17.0 & 5.44 & 5.38 & 5.41 \\
\hline 18.0 & 5.44 & 5.39 & 5.415 \\
\hline 19.0 & 5.44 & 5.35 & 5.395 \\
\hline 20.0 & 5.44 & 5.35 & 5.395 \\
\hline 21.0 & 5.51 & 5.45 & 5.48 \\
\hline 22.0 & 5.45 & 5.38 & 5.415 \\
\hline 23.0 & 5.44 & 5.37 & 5.405 \\
\hline 24.0 & 5.44 & 5.35 & 5.395 \\
\hline 25.0 & 5.44 & 5.4 & 5.42 \\
\hline
\end{tabular}

Fig.13.Table that includes data of Cs- 137 for reproducibility test in the pdf file.

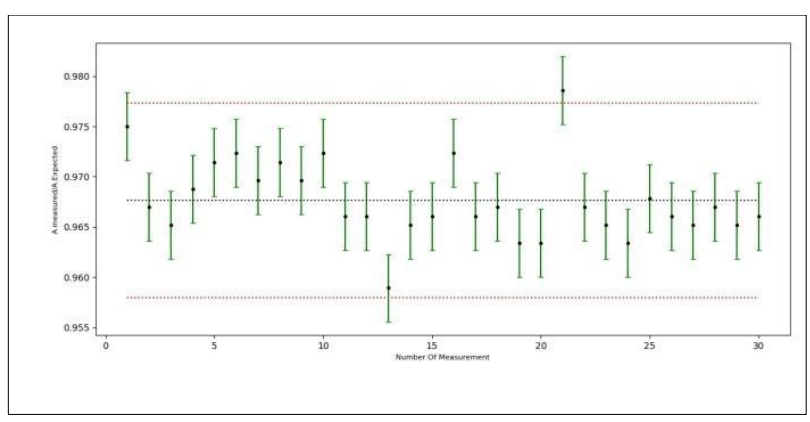

Fig.14.Control chart of Cs-137 for reproducibility test plotted with the program.

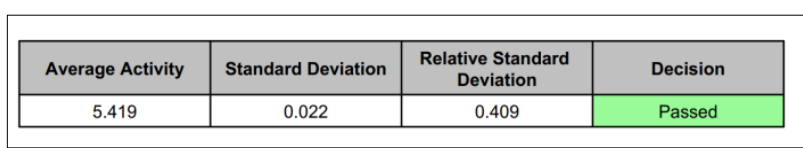

Fig.15.Table that includes results of reproducibility test in the pdf file.

Results of quality control tests of MEDI404 using this GUI confirm the robustness and the accuracy of the program since this one gives the same results provided using Excel 2010. However, it is not possible to estimate the uncertainty associated with measurements of a particular dose calibrator and to evaluate the instrument response without referencing to the experiment. Therefore, further work will be carried out to redress this lack. Monte Carlo method will be integrated into the GUI to estimate uncertainty. Furthermore, FLUKA Monte Carlo code will be merged with the GUI to simulate the response of DC and to assess sources of error and calculate correction factors that should be applied.

\section{Conclusion}


An effective performance testing process is critical to assure the accuracy of results provided by dose calibrators. This work offered a reliable and convenient GUI that enables to automate carrying out the major quality control tests of dose calibrators. The working process of this tool which is built using python 3.8 is described in this paper as well as results provided by the program of tests for MEDI404. Further work will attempt to cover sources of error that can affect a dose calibrator outcome and to establish a novel approach that avoids the complexity of determining correction factors using traceable sources.

\section{References}

[1] K. M. W.-R. Paul E. Christian, Nuclear Medicine and PET/CT, 7th Editio. Mosby, 2011.

[2] N. P. James E. Carey, Patrick Byrne, Larry DeWerd, Ralph Lieto, "The Selection, Use, Calibration, and Quality Assurance of Radionuclide Calibrators," 2012.

[3] A. R. Correia et al., "Volume corrections factors in the measurement of $99 \mathrm{mTc}$ and $123 \mathrm{I}$ activities in radionuclide calibrators," Radiol. Bras., vol. 45, no. 2, pp. 93-97, 2012, doi: 10.1590/s010039842012000200006 .

[4] L. Strigari et al., "Comparison of methods to determine accurate dose calibrator activity measurements," $J$. Exp. Clin. Cancer Res., vol. 27, no. 1, pp. 1-6, 2008, doi: 10.1186/1756-9966-27-14.

[5] V. Olšovcová and M. Havelka, "Monte Carlo calculations of calibration and geometry correction factors of a radionuclide calibrator," Appl. Radiat. Isot., vol. 64, no. 10-11, pp. 1370-1374, 2006, doi: 10.1016/j.apradiso.2006.02.047

[6] A. Ceccatelli et al., "Experimental determination of calibration settings of a commercially available radionuclide calibrator for various clinical measurement geometries and radionuclides," Appl. Radiat. Isot., vol. 65, no. 1, pp. 120-125, 2007, doi: 10.1016/j.apradiso.2006.06.009.

[7] M. Bauwens et al., "A comparison of four radionuclide dose calibrators using various radionuclides and measurement geometries clinically used in nuclear medicine," Phys. Medica, vol. 60, pp. 14-21, Apr. 2019, doi: 10.1016/j.ejmp.2019.03.012.

[8] S. Sharma, B. Singh, A. Koul, and B. R. Mittal, "Deviation in the predefined calibration factors of the dose calibrators and the associated inaccuracy in the radioactivity measurements of beta-gamma emitters," Indian J. Nucl. Med., vol. 30, no. 2, pp. 122-127, 2015, doi: 10.4103/0972-3919.152972.

[9] J. F. Valley, S. Bulling, M. Leresche, and C. Wastiel, "Determination of the efficiency of commercially available dose calibrators for beta-emitters.," J. Nucl. Med. Technol., vol. 31, no. 1, pp. 27-32, 2003.

[10] J. A. M. Santos, M. F. Carrasco, J. Lencart, and A. L. Bastos, "Syringe shape and positioning relative to efficiency volume inside dose calibrators and its role in nuclear medicine quality assurance programs," Appl. Radiat. Isot., vol. 67, no. 6, pp. 1104-1109, Jun. 2009, doi: 10.1016/j.apradiso.2009.01.084.

[11] International Commission on Radiological Protection,
"RADIATION PROTECTION PUBLISHED FOR The International Commission on Radiological Protection BY PERGAMON PRESS OXFORD NEW YORK TORONTO SYDNEY · FRANKFURT," 1977.

[12] D. L. Bailey, J. L. Humm, A. Todd-Pokropek, and A. van Aswegen, "Nuclear Medicine Physics. A Handbook for Teachers and Students.," Med. Phys., vol. 38 , no. 8, 2014.

[13] International Atomic Energy Agency, QUALITY ASSURANCE FOR RADIOACTIVITY MEASUREMENT IN NUCLEAR MEDICINE. IAEA, 2006.

[14] L. Cea, "Guide d 'utilisation et de contrôle qualité des activimètres," 2006.

[15] COMERCER, VDC-606 manual. 2014.

[16] Z. F. Gadd R, Baker M, Nijran KS, Owens S, Thomas W, Woods MJ, "Protocol for Establishing and Maintaining the Calibration of Medical Radionuclide Calibrators and their Quality Control Guide No. 93," 2006. 